\title{
A Case of Focal Choroidal Excavation Development Associated with Multiple Evanescent White Dot Syndrome
}

\author{
Hisashi Matsubara ${ }^{a}$ Eriko Uchiyama ${ }^{a}$ Katsuya Suzuki ${ }^{b}$ \\ Yoshito Matsuda ${ }^{\text {b, c }}$ Mineo Kondo ${ }^{a}$ \\ aDepartment of Ophthalmology, Mie University Graduate School of Medicine, Tsu, Japan; \\ ${ }^{b}$ Department of Ophthalmology and Visual Science, Nagoya City University Graduate \\ School of Medical Sciences, Nagoya, Japan; 'Department of Ophthalmology, \\ Komonokousei Hospital, Mie, Japan
}

\section{Keywords}

Choroidal excavation - Choroidal thickness - Multiple evanescent white dot syndrome . Optical coherence tomography

\begin{abstract}
Focal choroidal excavation (FCE) is described as an excavated lesion of the choroid that can be detected by optical coherence tomography (OCT). While the exact pathogenesis of FCE remains unclear, it has been proposed in some cases that there is an association with the inflammation in the outer retina. We present a case of FCE development that was detected by spectral domain OCT (SD-OCT) and found to be associated with multiple evanescent white dot syndrome (MEWDS). A 40-year-old Japanese woman was diagnosed with MEWDS based on multiple white dots observed from the posterior pole to the midperiphery, along with yellow granularity in the fovea. SD-OCT revealed separation between the retinal pigment epithelium (RPE) and Bruch's membrane (BM) and discontinuations of the ellipsoid zone, RPE, and BM. At 4 weeks after onset, several of the white dots disappeared, the yellow granularity in the fovea became small, and we detected nonconforming choroidal excavation under the central fovea. The choroidal excavation gradually deepened and changed to a conforming pattern. These findings suggest that the degree of the impairment caused by inflammation and the plasticity of the BM and RPE complex may be associated with different types of acquired FCE.
\end{abstract}




\section{Introduction}

Multiple evanescent white dot syndrome (MEWDS) is an unidentified chorioretinal disease, with multiple small subretinal white spots that extend from the posterior pole to the midperiphery [1]. Although the exact pathogenesis remains unclear, it has been proposed that the inflammation on the outer retina and the choroidal circulation disorder are caused by a viral infection. In fact, optical coherence tomography (OCT) has shown that there is a loss of the ellipsoid zone (EZ) and interdigitation zone [2]. Furthermore, indocyanine green angiography (ICGA) has demonstrated the presence of multiple hypofluorescence lesions in eyes with MEWDS [3]. Multimodal imaging results have suggested that the outer retina and choriocapillaris are damaged by inflammation [4] with this damage mediating the visual function impairment. On the other hand, focal choroidal excavation (FCE) has been described as an excavated lesion of the choroid that can be detected by OCT [1, 5, 6]. FCE has been further classified into conforming and nonconforming types based on the absence or presence of a separation between the neural retina and the retinal pigment epithelium (RPE) [6]. While the exact pathogenesis of FCE remains unclear, many studies have subsequently noted that FCE is associated with congenital or acquired choriocapillary abnormalities, including relationships between congenital choroidal abnormalities and developmental defects [6], inflammatory diseases [7-9], and choroidal vascular disorders [10]. However, only a few reports have described the detailed clinical course of the formation and development of FCE. In the present study, we report on the clinical course of a case of acquired FCE development associated with MEWDS detected by spectral domain OCT (SD-OCT).

\section{Case Report}

A 40-year-old Japanese woman presented with a complaint of blurred vision of her left eye for 1 week after catching a cold. Her past medical history was unremarkable. At the initial examination in our hospital, best-corrected visual acuity (BCVA) using a decimal visual test chart was 1.2 and 0.6 in her right and left eyes, while the intraocular pressure (IOP) was 16 and $13 \mathrm{~mm} \mathrm{Hg}$ in her right and left eyes, respectively. Slit-lamp examination showed there were no inflammatory cells in the anterior chamber and 1+ cells in the vitreous of her left eye. Ophthalmoscopy revealed multiple white dots ranging from the posterior pole to the midperiphery, with yellow granularity observed in the fovea (Fig. 1a). Fluorescein angiography (FA) revealed the presence of multiple hyperfluorescent spots from the early through the late phase, with staining of the optic disc observed in the late phase (Fig. 1b). ICGA demonstrated the presence of hypofluorescence in the fovea with multiple hypofluorescent spots ranging from the posterior pole to the midperiphery in the late phase (Fig. 1c). Hyperfluorescent lesions in FA and hypofluorescent lesions in ICGA demonstrated the presence of multiple white dots in the fundus. A Humphrey field analyzer revealed a slight enlargement of the blind spot of Mariotte in the left eye. SD-OCT demonstrated the presence of a separation between the RPE and Bruch's membrane (BM) (pigment epithelium detachment), discontinuations in the RPE and BM (Fig. 2a), and a hyper-reflective lesion in the outer retina under the fovea (Fig. 2a). A loss of the EZ (Fig. 2a) and a hyper-reflective lesion in the ganglion cell layer (Fig. 2a) were observed at the parafovea. Choroidal thicknesses at the center of the fovea and the edge of the pigment epithelium detachment were 235 and $250 \mu \mathrm{m}$, respectively (Fig. 2a). Three weeks after the onset, the hyper-reflection spread to the area between the inner lesion of the outer nuclear layer (Fig. 2b), and an abnormal hyper-reflective lesion appeared within the 
choroid (Fig. 2b). In addition, the separation between the RPE and BM was found to have almost completely disappeared (Fig. 2b). A hyper-reflective band was clearly observed from the RPE to the sclera under the fovea (Fig. 2b). The patient was diagnosed with MEWDS and treated with prednisolone, with the $15 \mathrm{mg}$ dose gradually tapered after the initial presentation. Four weeks after the onset, several of the white dots disappeared, the yellow granularity in the fovea became slightly smaller, and the BCVA in the left eye improved to 1.2, which was subsequently maintained. OCT showed there was a slight but clear choroidal excavation with a slight separation between the RPE and neural retina detected under the central fovea (Fig. 2c). Choroidal thicknesses at the center of the fovea and at the edge of the FCE were 183 and $228 \mu \mathrm{m}$, respectively (Fig. 2c). Three months after the onset, the subretinal fluid had been completely absorbed, with the nonconforming FCE then changing to the conforming FCE (Fig. $2 \mathrm{~d}$ ). BCVA in the left eye was 1.2, a value that was subsequently maintained. The hyper-reflective lesion in the ganglion cell layer completely resolved. Choroidal thicknesses at the center of the fovea and at the edge of the FCE decreased and became 147 and $200 \mu \mathrm{m}$, respectively (Fig. 2d). Six months after the onset, BCVA of the left eye remained at 1.2 and the EZ at the fovea was clearly detected. There has been no subsequent recurrence of MEWDS or choroidal neovascularization observed. The choroidal excavation has gradually deepened (Fig. 2e), with the choroidal thicknesses at the center of the fovea and at the edge of the FCE determined to be 132 and $191 \mu \mathrm{m}$, respectively (Fig. 2e).

\section{Discussion}

Although many researchers have proposed various pathologies for FCE, the exact pathology of FCE remains unclear. Recently, it was reported that FCE was only detected in 3 out of 1,697 eyes of children and young adults, with this low prevalence suggesting that most FCE may be acquired [11]. However, there have been a few studies that have reported on the developmental process of acquired FCE. Hashimoto et al. [7] reported that OCT was able to show a sequential progression of a conforming-type FCE secondary to MEWDS. Furthermore, these authors proposed that active inflammation between the outer retina and the inner choroid impaired the RPE and BM, with the outer retina and choroidal tissues adhering through the RPE/BM ruptures. In addition, the IOP caused photoreceptor protrusion through the RPE/BM ruptures into the choroid, with contraction of the fibrotic lesions then drawing the overlying retinal tissues backward. Moreover, when nonconforming FCE is present, the absence of adhesion between the neural retina and the RPE helps to keep the photoreceptor tips in place [7]. In the current case, RPE and BM separation and hyper-reflection were detected in the outer retina. These findings suggest that there was localized inflammation in the outer retina and inner choroid, similar to that which has been reported in a previous case. However, there are some differences between the current and previous cases. First, although it was reported that the choroidal thickness increased during the acute phase in MEWDS [4], we found that there was little increase in the choroidal thickness at the fovea and parafovea at the initial visit. Second, the yellow white granularity lesion was narrow, and the intensity of hyperfluorescence for the FA was weak. Finally, the discontinuations of the RPE and BM were milder, with the abnormal hyper-reflective lesion disappearing in the choroid at 4 weeks after onset. These differences suggest that the inflammation in the current case might be milder than that reported for the previous case, and as a result, since the BM and RPE disorder was also milder, the photoreceptor and choroid adhesion did not occur. Histologically, as BM is constructed of elastic and collagen fibers, this means that BM has plasticity and the ability to stretch or return 
to its original shape depending on the IOP and choroidal blood volume changes. However, since we detected a hyper-reflective band that was present up through the sclera at 3 weeks after the onset, this suggests that there was a structural disorder in the BM and RPE complex. Therefore, a change in the plasticity of the BM and RPE complex might be associated with the FCE development in our current case. Based on our current findings, we developed the following hypotheses regarding the mechanism of FCE development (Fig. 3). Mild inflammation might cause the BM and RPE disorder, and depending on the intensity, the BM and RPE might become weaker. Thus, upon improvement of the inflammation, there is a gradual decrease in the choroidal thickness that can lead to a decrease in the pressure that pushes the BM and RPE complex towards the vitreous. On the other hand, the IOP, which has the ability to push the $\mathrm{BM}$ and RPE complex towards the choroid, did not change. These changes occurred due to the disproportion of the pressure to the impaired BM and RPE complex. As a result, this causes the BM and RPE complex to fall towards the choroid, thereby leading to the formation of a nonconforming FCE. In the recovery of RPE function, the subretinal fluid is absorbed, which causes the nonconforming FCE to conform.

\section{Conclusions}

In conclusion, the observation of a case of choroidal excavation formation in MEWDS suggests that clinicians need to be made aware of this possible complication. Multimodal image findings for the current case demonstrate that the degree of the impairment caused by the inflammation and the plasticity of the BM and RPE complex might be associated with differences in the types of acquired FCE.

\section{Statement of Ethics}

The patient provided written informed consent for submission of the case report and any accompanying images.

This study was approved by the Ethics Committee of Mie University Graduate School of Medicine, Tsu City, Mie, Japan.

\section{Disclosure Statement}

The authors declare that they have no conflicts of interest.

\section{References}

1 Jampol LM, Sieving PA, Pugh D, Fishman GA, Gilbert H. Multiple evanescent white dot syndrome. I. Clinical findings. Arch Ophthalmol. 1984 May;102(5):671-4.

2 Li D, Kishi S. Restored photoreceptor outer segment damage in multiple evanescent white dot syndrome. Ophthalmology. 2009 Apr;116(4):762-70.

3 Ie D, Glaser BM, Murphy RP, Gordon LW, Sjaarda RN, Thompson JT. Indocyanine green angiography in multiple evanescent white-dot syndrome. Am J Ophthalmol. 1994 Jan;117(1):7-12.

4 Aoyagi R, Hayashi T, Masai A, Mitooka K, Gekka T, Kozaki K, et al. Subfoveal choroidal thickn ess in multiple evanescent white dot syndrome. Clin Exp Optom. 2012 Mar;95(2):212-7.

5 Jampol LM, Shankle J, Schroeder R, Tornambe P, Spaide RF, Hee MR. Diagnostic and therapeutic challenges. Retina. 2006 Nov-Dec;26(9):1072-6. 


\section{Case Reports in Ophthalmology}

\begin{tabular}{l|l}
\hline DOI: 10.1159/000492747 & (c) 2018 The Author(s). Published by S. Karger AG, Basel \\
\hline
\end{tabular} www.karger.com/cop

Matsubara et al.: A Case of Focal Choroidal Excavation Development Associated with Multiple Evanescent White Dot Syndrome

6 Margolis R, Mukkamala SK, Jampol LM, Spaide RF, Ober MD, Sorenson JA, et al. The expanded spectrum of focal choroidal excavation. Arch Ophthalmol. 2011 Oct;129(10):1320-5.

7 Hashimoto Y, Saito W, Noda K, Ishida S. Acquired focal choroidal excavation associated with multiple evanescent white dot syndrome: observations at onset and a pathogenic hypothesis. BMC Ophthalmol. 2014 Nov;14(1):135.

8 Hashida N, Fok A, Nishida K. Choroidal excavation in vogt-koyanagi-harada disease. Case Rep Ophthalmol. 2014 Jul;5(2):222-5.

9 Jabbarpoor Bonyadi MH, Hassanpour K, Soheilian M. Recurrent focal choroidal excavation following multiple evanescent white dot syndrome (MEWDS) associated with acute idiopathic blind spot enlargement. Int Ophthalmol. 2018 Apr;38(2):815-21.

10 Wakabayashi Y, Nishimura A, Higashide T, Ijiri S, Sugiyama K. Unilateral choroidal excavation in the macula detected by spectral-domain optical coherence tomography. Acta Ophthalmol. 2010 May;88(3):e87-91.

11 Park KA, Oh SY. The absence of focal choroidal excavation in children and adolescents without retinal or choroidal disorders or ocular trauma. Eye (Lond). 2015 Jun;29(6):841-2.
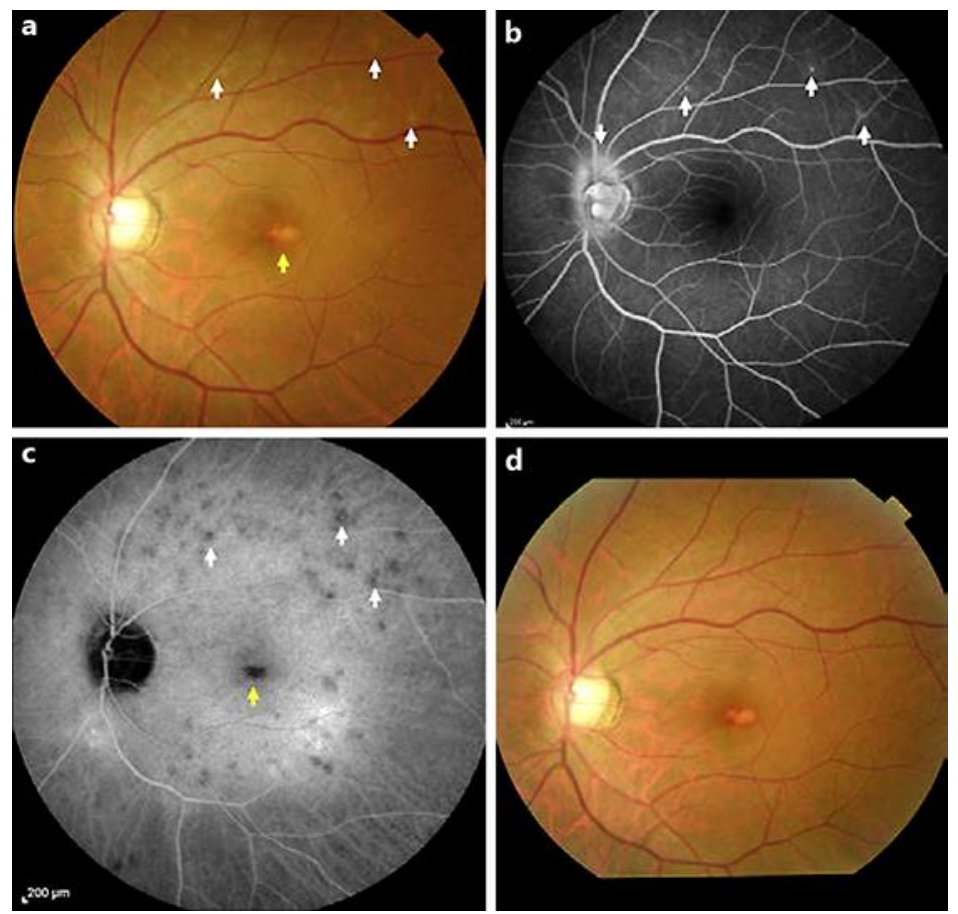

Fig. 1. Fundus photograph, fluorescein and indocyanine green angiography images of the left eye with multiple evanescent white dot syndrome (MEWDS) at the initial visit (a, b, c) and at 3 months after onset (d) Multiple white dots (white arrows) are observed from the posterior pole to the midperiphery, with yellow granularity in the fovea (yellow arrows). Late-phase fluorescein angiography revealed that multiple hyperfluorescent spots (white arrows) were present from the early to the late phase, along with staining of the optic disc (white arrows). Indocyanine green angiography showed that the fovea was hypofluorescent (yellow arrows), with multiple hypofluorescent spots observed from the posterior pole to the midperiphery in the late phase (white arrows). White dots disappeared and the edge of the yellow granularity in the fovea became clear. 


\section{Case Reports in Ophthalmology}
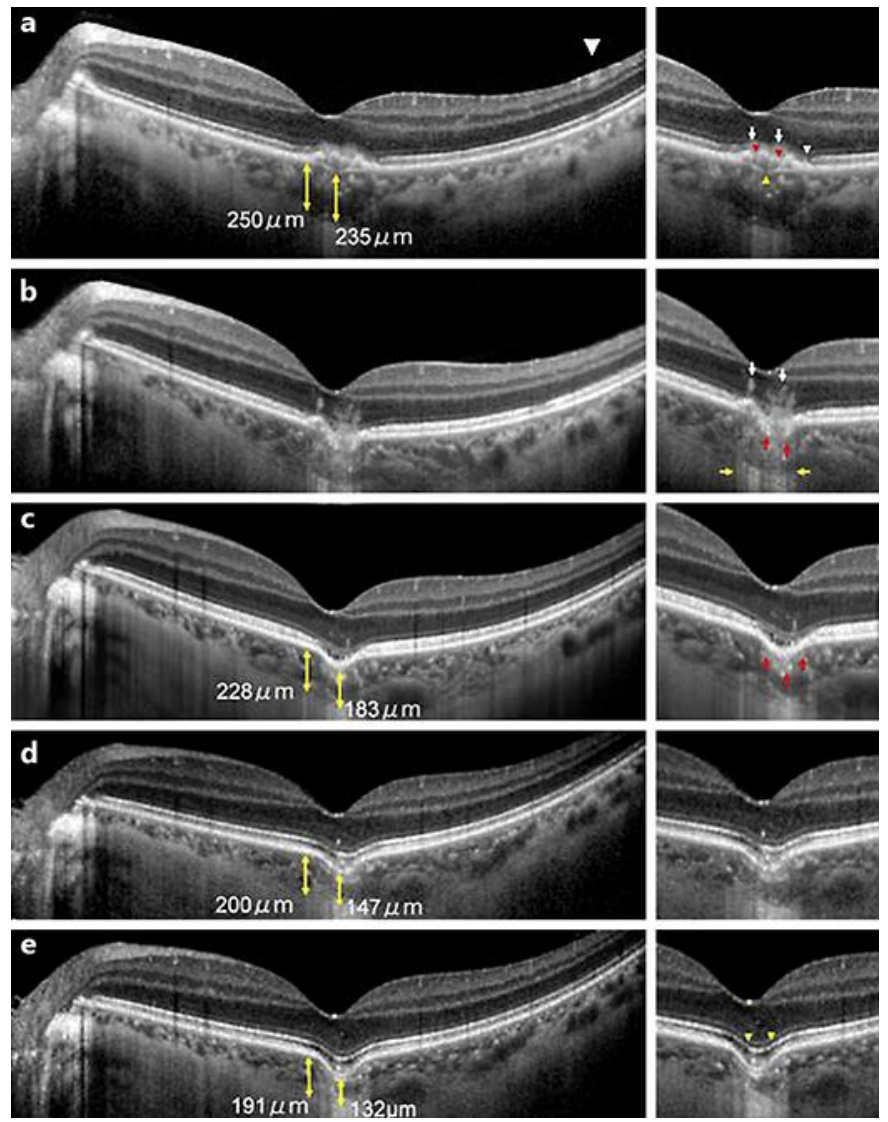

Fig. 2. Spectral domain optical coherence tomographic (SD-OCT) images of the left eye. At the initial visit, SD-OCT images demonstrated that there was a separation between the retinal pigment epithelium (RPE) and Bruch's membrane (BM) (pigment epithelium detachment), discontinuations of the RPE and BM (red and yellow arrowheads), and the presence of a hyper-reflective lesion in the outer retina under the fovea (white arrows). The loss of the ellipsoid zone (EZ) (white arrowheads) and the hyper-reflective lesion in the ganglion cell layer (large white arrowhead in the left panel) were observed in the parafovea. At 3 weeks after the onset, the hyper-reflection spread to the area of the inner lesion of the outer nuclear layer (white arrows), there was an abnormal hyper-reflective lesion appearing within the choroid (red arrows), with the separation between the RPE and BM almost completely disappearing. The hyper-reflective band was clearly observed from the RPE to the sclera under the fovea (yellow arrows). At 4 weeks after the onset, OCT detected the choroidal excavation with a slight separation between the RPE and neural retina under the central fovea (red arrows). At 3 months after the onset, subretinal fluid was completely absorbed, with the nonconforming focal choroidal excavation (FCE) changing to the conforming FCE. At 6 months after the onset, the EZ at the fovea was clearly detected (yellow arrowheads). There was a gradual deepening of the choroidal excavation. 


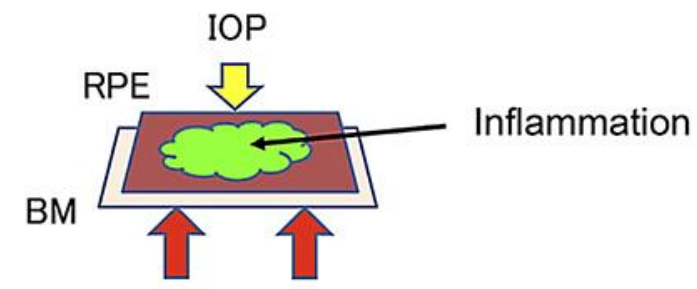

a

Choroidal pressure

b



c


RPE and BM herniation

\section{d Decrease of choroidal pressure}

Fig. 3. Hypothesis regarding the mechanism of focal choroidal excavation (FCE) formation in the present case. Initially, inflammation in the outer retina, retinal pigment epithelium (RPE), and Bruch's membrane (BM) occurs. At the same time, there is an increase in the choroidal exudation and thickness and in the choroidal pressure. Subsequently, the RPE and BM separate and pigment epithelium detachment (PED) forms. The balance of the pressure to BM between the intraocular pressure (IOP) and choroidal pressure is maintained. As the inflammation causes impairment of the RPE and BM, they become weaker. After improvement of the inflammation, there is a decrease in the choroidal exudation and pressure. If the pressure to the RPE and BM is disproportionate, the BM and RPE herniate towards the choroid. 